\title{
Les sites de réseautage professionnels au service du marketing : le cas Viadeo. Entrevue avec Olivier Fecherolle
}

Entretien réalisé par Thomas Stenger et Alexandre Coutant

Thomas Stenger et Alexandre Coutant

\section{(2) OpenEdition}

Édition électronique

URL : http://journals.openedition.org/communicationorganisation/4268

DOI : 10.4000/communicationorganisation.4268

ISSN : $1775-3546$

Éditeur

Presses universitaires de Bordeaux

Édition imprimée

Date de publication : 1 juin 2013

Pagination : 283-289

ISBN : 978-2-86781-883-7

ISSN : $1168-5549$

\section{Référence électronique}

Thomas Stenger et Alexandre Coutant, «Les sites de réseautage professionnels au service du marketing : le cas Viadeo. Entrevue avec Olivier Fecherolle », Communication et organisation [En ligne], 43 | 2013, mis en ligne le 01 juin 2015, consulté le 04 mai 2019. URL : http://journals.openedition.org/ communicationorganisation/4268 ; DOI : 10.4000/communicationorganisation.4268 


\section{Entretien réalisé par Thomas STENGER ${ }^{1}$ et Alexandre COUTANT ${ }^{2}$}

\section{Les sites de réseautage professionnels au service du marketing : le cas Viadeo. Entrevue avec Olivier Fecherolle}

Olivier Fecherolle est Directeur Général de Viadeo France et responsable Marketing Europe. Viadeo est un site de réseautage ${ }^{3}$ professionnel fondé en 2004 qui " réunit plus de 50 millions de professionnels ». Cette société française qui compte aujourd'hui 400 salariés s'est très vite internationalisée. Elle est présente en Europe du sud - France, Italie, Espagne, Portugal -, en Grande-Bretagne et, par une stratégie de croissance externe, dans les pays émergents : Chine, Inde, Mexique et Amérique du Sud à travers des sites nationaux (Tienji.com en Chine, ApnaCircle.com en Inde ${ }^{4}$ ). Si ce site de réseautage professionnel (ou networking) a longtemps été perçu comme une plateforme dédiée à l'emploi, Olivier Fecherolle, DG et responsable marketing Europe nous explique qu'il s'agit au moins autant d'une formidable base de données pour le marketing qui ne demande qu'à être exploitée comme telle par les marques, en particulier pour le développement de relations BtoB.

\section{Pouvez-vous présenter Viadeo et son modèle d'affaires?}

Tous les lecteurs doivent déjà connaître et j'espère être inscrits sur Viadeo ! Je vais donc tout de suite vous parler de notre business model. Il est assez équilibré, par rapport à d'autres réseaux socionumériques, avec d'un côté des abonnements premiums qui représentent $50 \%$ des revenus. Ici nous nous appuyons sur un modèle freemium ${ }^{5}$ typique de l'économie Internet d'aujourd'hui : une inscription gratuite, un premier niveau d'usage gratuit et un niveau d'usage supérieur avec fonctionnalités réservées aux membres qui souscrivent un abonnement payant. D'un autre côté, $50 \%$ des revenus proviennent du $B$ to $B$ avec d'une part, la publicité -nous disposons d'une régie intégrée à destination des annonceurs - et d'autre part, les recruteurs. Nous proposons la publication d'offres d'emplois et l'identification de

\footnotetext{
1 Maître de conférences, IAE de Poitiers, laboratoire CEREGE et ISCC; stenger@iae.univ-poitiers.fr 2 Maître de conférences, Université de Franche-Comté, ELLIADD, équipe OUN ; coutant.alexandre@gmail.com 3 Nous utilisons le terme réseautage comme traduction de l'anglais Social networking site, qui permet de les distinguer des réseaux socionumériques, Social network site.

4 Voir également : Fecherolle O. (2011), «Viadeo : réseaux sociaux et networking », Hermès, 59, CNRS Ed., p. 143-146 et les informations officielles fournies par Viadeo sur http://corporate.viadeo.com

5 Freemium : néologisme provenant de la contraction de « free » et de " premium ».
} 
profils, de talents, de compétences sur la base de données Viadeo. À titre de comparaison, les revenus de LinkedIn proviendraient essentiellement d'abonnements des membres et des recruteurs.

\section{Vous ne présentez pas Viadeo comme une plateforme d'emploi. Votre image est pourtant très liée aux $\mathrm{CV}$, au recrutement...}

Nous sommes fiers d'être perçus comme un outil d'aide à l'emploi, mais il ne faut pas nous réduire à ça ! Nous sommes ravis de le faire, et je pense que nous offrons un service qui est très performant à ce niveau, mais c'est réducteur de nous définir ainsi. C'est peut-être dû au fait que les services $\mathrm{RH}$ ont tout de suite vu l'opportunité que représentait Viadeo. Ils ont été les premiers à venir nous voir et à nous dire : votre outil est magnifique, avec votre média on va trouver des candidats. Du coup, on a développé des outils RH en premier.

\section{Les marketeurs sont un peu à la traîne alors ?}

Plus maintenant. Aujourd'hui Viadeo, c'est d'abord une plateforme pour de la prospection, pour trouver des clients et échanger avec eux. La partie $\mathrm{RH}$ est désormais secondaire et l'essentiel de notre activité provient de ces relations B2B. Mais ce sont surtout les RH, puis les commerciaux qui ont démarré et les marketeurs ont mis plus de temps pour s'approprier la plateforme. Peut-être aussi n'avions nous pas forcément les bons outils marketing à leur proposer au départ.

Les outils marketing justement... Viadeo propose un système de publicité bien sûr, d'e-mailing, des pages, des groupes / hubs... Pouvez-vous nous éclairer?

Pour la publicité en ligne, le modèle classique, c'est Google qui l'a inventé. C'est-à-dire que, si je suis une marque, j'attends que quelqu'un tape mon nom ou des mots clés dans Google pour lui dire que j’existe en lui proposant des liens hypertextes. Avec Viadeo, c'est le cheminement inverse. Par exemple : vous êtes une société d'informatique et vous lancez un nouveau serveur; nous allons directement cibler des entreprises de plus de 100 personnes dans le domaine « informatique ». Quand les membres de Viadeo se connectent, ils verront dans leur « weekly report » reçu par mail, ou directement sur le site, que cette entreprise propose un nouveau serveur. Vous voyez : la démarche est inversée. C'est l'information qui vient vers moi. Nous ciblons d'abord puis affichons la publicité ensuite. Et nous sommes capables d'aller très loin avec le ciblage. Naturellement, plus vous ciblez, plus le contact est cher, mais il vaut mieux payer des contacts hyper qualifiés! On peut par exemple, toucher les membres qui sont inscrits dans les hubs/groupes qui parlent d'automobile, d'informatique... qui ont lu ou partagé des articles consacrés à tel thème. Viadeo est donc une plateforme qui permet de faire un ciblage extrêmement fin et de proposer des campagnes de communication pour les annonceurs. 
Encadré 1 - Parlez-nous de la campagne de Fiat sur Viadeo, c'est un cas intéressant

Oui, on avait travaillé le ciblage à partir d'un profil d'acheteur type pour un modèle spécifique de Fiat (Punto). Ensuite, cela passe par de la publicité et la création d'un formulaire en ligne de prises de rendez-vous. Puis le contact est invité à se rendre chez le concessionnaire le plus proche du domicile pour essayer le véhicule. Fiat a pu mesurer très précisément le nombre de formulaires remplis, le nombre de visites en concession, le nombre d'essais et enfin de voitures vendues. C'est une opération extrêmement bien menée car évaluée du début à la fin.

Le ciblage publicitaire repose donc sur les informations renseignées sur les profils, alors que Google Adwords a une approche comportementale, en s'appuyant sur les requêtes des internautes...

Oui, c'est une de nos forces, nous vous donnons accès aux professionnels que vous cherchez à contacter même si son comportement ne laisse pas penser qu'il peut vous intéresser. Nous pouvons proposer de cibler peut-être seulement 500 ou 1000 personnes mais de façon très qualifiée par rapport à ce qui est pratiqué sur le web. Sinon, pour la tarification on fonctionnait en CPM (i.e. coût pour mille) et on est aussi passé au coût par clic (CPC). Pour les PME, nous avons aussi une autre offre "Social Ads» qui permet pour quelques centaines d'euros de bénéficier de la viralité de Viadeo. Le principe : si votre annonce "fonctionne ", c'est-à-dire si les membres qui la voient cliquent dessus, nous offrons des affichages gratuits vous permettant d'une part de baisser le coût de cette campagne mais aussi de la rendre virale.

\section{Encadré 2 : Social ads sur Viadeo}

Lancé en mai 2011, le service « social ads ${ }^{6}$ permet d'effectuer la promotion d'un profil ou d'un site Web directement au sein de la plateforme. Son principe est original : plus la publicité a de succès, plus elle sera diffusée. Chaque clic rapporte 100 affichages supplémentaires. Le coût de la campagne correspond ainsi au nombre de contacts auprès desquels diffuser l'annonce le premier jour, les jours suivants ne constituant que des bonus et ce pendant une semaine. Les contraintes de création sont strictes : l'annonce, modifiable gratuitement, ne doit pas dépasser les 140 caractères (auxquels on ajoute un titre de 26 caractères ainsi qu'une illustration). Le ciblage par défaut est national, mais des options (payantes) permettent de préciser celui-ci par région, domaine d'activités ou fonction occupée. 


\section{Parlez-nous des groupes / hubs Corporate. Comment les utiliser?}

Les « hubs Corporate » Viadeo ont été lancés en 2010 ; ce sont des groupes, initiés non pas par des membres comme c'est souvent le cas sur les réseaux socionumériques, mais par les marques elles-mêmes, avec une volonté d'animation marketing : dans une logique de communauté de marque en fait. Il s'agit de recruter, d'animer et d'échanger avec les membres de cette communauté. Les hubs de Sogeti, d'IBM sont des exemples intéressants de communautés de professionnels qui les utilisent pour entretenir une relation différente avec leur cible sur les réseaux socionumériques. Ces hubs sponsorisés par des marques sont en quelque sorte des forums de discussion auxquels on peut ajouter des outils tels que les livres blancs à télécharger, lorsqu'on veut diffuser un contenu, créer du lead ${ }^{7}$, de la communication online, etc.

\section{Que diriez-vous à une marque qui hésite entre créer une page sur Facebook et un hub sur Viadeo?}

Ce sont deux choses complètement indépendantes. C'est-à-dire qu'une page sur Facebook, c'est davantage pour entretenir la relation client BtoC. Si on s'appelle Danone, c'est sûr qu'il vaut mieux créer une page Facebook, afin de mettre en avant ses produits, ses films publicitaires, créer une dynamique... Starbucks, Danone, Red Bull etc. ce sont des marques grand public. Sur Viadeo on retrouve davantage des marques à vocation $B$ to $B$ : Banque Populaire, Hewlett-Packard, IBM, Manpower... Ce sont donc deux approches distinctes et complémentaires. Je conseillerais même de créer les deux selon les objectifs de la marque.

\section{Encadré 3 : Viadeo et Facebook, questions d'usages et de profils}

Les plateformes Viadeo et Facebook sont assez proches techniquement mais elles diffèrent largement du point de vue des usages. Viadeo revendique une finalité professionnelle et cherche à mettre en réseau "collègues, collaborateurs, experts, prospects et client » (cf. page d'accueil). Facebook est avant tout une plateforme où se retrouvent les proches, les amis, les «personnes qui comptent dans votre vie » (idem). Les opportunités marketing dépendent fortement de ces usages et des normes d'interaction associées qui renvoient respectivement à la sphère professionnelle et aux relations entre entreprises (Viadeo) et à la sociabilité, l'amitié, et le divertissement (Facebook). Le nombre d'utilisateurs dans le monde (40 millions / 850 millions) comme en France ( 5 millions / 25 millions) reflète cette dichotomie entre professionnels et 4 grand public ${ }^{8}$.

7 Le terme « lead ", employé en e-marketing, marketing direct (ainsi qu'en vente) peut revêtir différentes significations. Il désigne généralement un nouveau prospect qualifié, obtenu grâce à une action marketing. 8 Pour une distinction entre réseaux socionumériques et réseaux sociaux professionnels, voir Stenger T., Coutant A. (2013), "Une cartographie des médias sociaux - Pour une approche sociotechnique », Décisions 
Le type de démarche marketing dépend également du type de données renseignées par les utilisateurs sur leur profil. Sur Facebook, les utilisateurs s'expriment très largement sur leurs loisirs, leurs préférences et goûts relatifs à plusieurs univers de consommation (cinéma, musique, sport, jeux vidéo, voyages...). Mais comme l'humour et le second degré sont au cœur de la culture socionumérique, la fiabilité des données doit être examinée avec prudence. À l'opposé, Viadeo dispose d'informations d'un haut niveau de fiabilité car les utilisateurs remplissent un profil destiné à des collaborateurs, prospects, clients et recruteurs. Ces informations concernent un ensemble bien plus restreint de sujets que sur Facebook. En revanche, elles permettent aisément d'associer les profils à des niveaux de revenus, diplôme, PCS et à des critères géographiques.

Comment mesurez-vous l'efficacité des campagnes de communication ? On parle de lead, de taux de clics, de ROI... Quels sont pour vous les bons indicateurs?

On lit parfois : " avec les réseaux sociaux, on ne peut pas mesurer le ROI " mais c'est faux. Nos offres sont assorties d'outils de mesure qui fonctionnent avec des critères désormais classiques, comme le taux de clics sur les produits publicitaires etc. Après, ce qui est plus difficile à évaluer, effectivement, c'est le changement d'image. Lorsque Sogeti veut travailler sur son image pour dire : voilà je suis Sogeti, filiale de Capgemini, je ne suis pas une machine à embaucher du consultant regardez, je suis aussi un expert sur tels sujets et vous pouvez venir discuter avec nous sur notre groupe Viadeo ${ }^{9}$, cela influence son image de la marque. C'est bien sûr plus délicat d'apprécier la modification d'une image de marque, suite à la présence d'une marque sur Viadeo, à l'animation d'un hub, à la publication de livres blancs... Je peux même dire qu'il y a une certaine frustration parce qu'on aimerait mieux mesurer la performance de ces actions. Toutefois, on peut évaluer l'engagement, le nombre de personnes contactées par la marque, le nombre de téléchargements d'un livre blanc, le nombre de personnes qui rejoignent un groupe, de contacts ayant répondu à l'e-mail, de contacts transformés, etc. On a désormais un certain nombre d'indicateurs. Et puis, parfois, comme pour Fiat (cf. encadré 1 ) on peut même aller jusqu'à mesurer le nombre d'essais et de ventes.

Marketing, 70 ; Stenger T., Coutant A. (coord.), (2011), Ces réseaux numériques dits sociaux, Hermès, 59, CNRS Éd.

9 Cf. http://www.viadeo.com/groups/sogeti : espace Sogeti dédié à « la rencontre et l'échange entre la communauté d'experts de Sogeti en France et ceux qui partagent avec nous de nombreux centres d'intérêt et d'expertise ». 
Les réseaux socionumériques sont de plus en plus envisagés comme des dispositifs permettant de développer un marketing local et des démarches de proximité...

Oui, c'est l'avantage du ciblage. Nous faisons par exemple beaucoup d'opérations avec les organisateurs de salon. Nous avons de très bons drivers parce qu'on est capable de cibler géographiquement, par domaine d'activité, par fonction et on peut calibrer l'envoi dans le temps. C'est-à-dire que si vous faites un salon sur l'e-marketing à Nantes, je peux vous dire qu'on est capable d'une manière très efficace de faire venir du monde au salon. Sur la population du département Loire-Atlantique, ou du Grand-Ouest si on veut avoir un recrutement plus large, nous ciblons les professions concernées en envoyant une publicité par un système d'alerte mail de la semaine moins 2 à la semaine 0 avant l'événement. Donc sur les campagnes locales, nous sommes très bien outillés.

La démarche repose donc sur une base de données très qualifiée, fondée sur les profils des membres, pourtant vous ne parlez jamais de marketing direct et d'e-mailing mais toujours de publicité...

Ce n'est pas de l'e-mailing parce qu'on ne cède pas nos données. Aucun annonceur n'a accès aux mails de nos membres. Mais c'est de la publicité embarquée sur un $e$-mail à destination des contact-cibles. Je n'aime pas le terme $e$-mailing parce qu'on a l'impression que l'annonceur repart avec les données de nos membres, et ça jamais ! On ne l'a jamais fait et on ne le fera jamais.

\section{Alors, justement quelles données sont accessibles aux entreprises?}

Nous ne cédons aucune donnée. Mais nous proposons aux entreprises de construire des fichiers avec les caractéristiques désirées. Dites-moi les profils que vous voulez atteindre, donnez-moi les caractéristiques les plus précises des membres que vous voulez atteindre et nous construisons la base de données pour vous. En plus des informations qu'ils donnent pour construire leur profil, nous pouvons aussi utiliser des données comportementales. Identifier par exemple les personnes qui se sont inscrites à un $b u b$ consacré à l'automobile, voire même celles qui auront lu ou partagé un article sur l'automobile... On peut aller très loin dans le ciblage. En pratique, aujourd'hui, nous sommes capables d'aller beaucoup plus loin que ce que les annonceurs nous demandent. On peut utiliser tellement de critères...

Pour conclure, quel est le principal intérêt de Viadeo pour la pratique du marketing?

Le ciblage ! C'est incontestable. Et le sérieux. Nous sommes un média social, dans un contexte professionnel, structuré en termes d'outils, de profils d'utilisateurs, de pratiques. Pratiques qui sont plus proches des 
habitudes de travail des directions marketing que d'autres médias sociaux plus fun... On dit parfois que Viadeo est trop sérieux, pas assez fun, qu'il y a moins de buzz chez nous. Oui, c'est sûr qu'il y a moins de buzz en volume que sur des plateformes plus grand public, mais nous répondons aussi qu'il n'y a pas de bad buzz et que chez nous le buzz est mieux ciblé ${ }^{10}$.

10 Pour un développement sur les relations entre marketing et médias sociaux, voir notamment : Vernette E., Tissier Desbordes E. (2012), Le repérage marketing des influenceurs dans les réseaux sociaux : des dangers de l'ignorance aux risques de l'à peu près, Décisions Marketing, 67, 5-9 ; Kaplan A., Haenlein M. (coord.), (2011), Recherche et Applications en Marketing, sept., 26, 3 ; Stenger T., Coutant A. (2011), «Web 2.0 et médias sociaux », p. 63-116 in Stenger T., Bourliataux-Lajoinie S., E-marketing et E-commerce, coll. Management Sup, Dunod. 
